\title{
Systematic Review \\ Tumor-Infiltrating Lymphocytes as Biomarkers of Treatment Response and Long-Term Survival in Patients with Rectal Cancer: A Systematic Review and Meta-Analysis
}

\author{
Adile Orhan ${ }^{1, *,+} \mathbb{D}$, Faisal Khesrawi ${ }^{1,+}{ }^{,}$, Michael Tvilling Madsen ${ }^{1} \mathbb{D}$, Rasmus Peuliche Vogelsang ${ }^{1}$, \\ Niclas Dohrn 1,2 ${ }^{\mathbb{D}}$, Anne-Marie Kanstrup Fiehn ${ }^{1,3,4} \mathbb{D}^{-}$and Ismail Gögenur 1,4
}

check for updates

Citation: Orhan, A.; Khesrawi, F.; Tvilling Madsen, M.; Peuliche Vogelsang, R.; Dohrn, N.; Kanstrup Fiehn, A.-M.; Gögenur, I.

Tumor-Infiltrating Lymphocytes as Biomarkers of Treatment Response and Long-Term Survival in Patients with Rectal Cancer: A Systematic Review and Meta-Analysis. Cancers 2022, 14, 636. https://doi.org/ 10.3390/cancers14030636 Academic Editor: Esteban Orenes Piñero

Received: 6 January 2022

Accepted: 25 January 2022

Published: 27 January 2022

Publisher's Note: MDPI stays neutral with regard to jurisdictional claims in published maps and institutional affiliations.

Copyright: (c) 2022 by the authors. Licensee MDPI, Basel, Switzerland. This article is an open access article distributed under the terms and conditions of the Creative Commons Attribution (CC BY) license (https:// creativecommons.org/licenses/by/ $4.0 /)$.
1 Center for Surgical Science, Zealand University Hospital, Lykkebaekvej 1, DK-4600 Køge, Denmark; faisalkhesrawi@gmail.com (F.K.); mitm@regionsjaelland.dk (M.T.M.); rvo@regionsjaelland.dk (R.P.V.); Niclas.Dohrn@regionh.dk (N.D.); ankf@regionsjaelland.dk (A.-M.K.F.); igo@regionsjaelland.dk (I.G.)

2 Department of Surgery, Copenhagen University Hospital, Herlev \& Gentofte, Borgmester Ib Juuls Vej 1, DK-2730 Herlev, Denmark

3 Department of Pathology, Zealand University Hospital, Sygehusvej 10, DK-4000 Roskilde, Denmark

4 Institute for Clinical Medicine, University of Copenhagen, Blegdamsvej 3b, DK-2200 Copenhagen, Denmark

* Correspondence: aor@regionsjaelland.dk

$+\quad$ These authors contributed equally to this work.

Simple Summary: This study investigated tumor-infiltrating lymphocytes (TILs) in pretherapeutic biopsies as biomarkers of treatment response and long-term prognosis in patients with locally advanced rectal cancer undergoing neoadjuvant chemoradiotherapy. A systematic review and metaanalysis was performed in accordance with the PRISMA guidelines. The results indicate that it is possible to identify a sub-group of patients with improved treatment response and long-term prognosis by assessing the density of $C D 8^{+}$TILs at the time of diagnosis.

Abstract: Neoadjuvant chemoradiotherapy (NCRT) is indicated in locally advanced rectal cancer (LARC) to downstage tumors before surgery. Watchful waiting may be a treatment option to avoid surgery in patients, obtaining a complete clinical response. However, biomarkers predictive of treatment response and long-term prognosis are lacking. Here we investigated tumor-infiltrating lymphocytes (TILs) in pretherapeutic biopsies as predictive and prognostic biomarkers. A systematic review and meta-analysis was performed in accordance with the PRISMA guidelines. In total, 429 articles were identified, of which 19 studies were included in the systematic review and 14 studies in the meta-analysis. Patients with high pretherapeutic CD8 ${ }^{+}$TILs density had an increased likelihood of achieving a pathological complete response ( $\mathrm{RR}=2.71 ; 95 \% \mathrm{CI}$ : 1.58-4.66) or a complete or nearcomplete pathological treatment response ( $R R=1.86$; 95\% CI: 1.50-2.29). Furthermore, high CD8 ${ }^{+}$ TILs density was a favorable prognostic factor for disease-free survival (HR $=0.57 ; 95 \% \mathrm{CI}: 0.38-0.86$ ) and overall survival ( $\mathrm{HR}=0.43 ; 95 \% \mathrm{CI}: 0.27-0.69) . \mathrm{CD}^{+}, \mathrm{CD}^{+}$, and $\mathrm{FOXP3}^{+}$TILs were not identified as predictive or prognostic biomarkers. Thus, assessing pretherapeutic CD8 ${ }^{+}$TILs density may assist in identifying patients with increased sensitivity to NCRT and favorable long-term prognosis.

Keywords: rectal neoplasms; tumor-infiltrating lymphocytes; cancer biomarkers; $\mathrm{CD}^{+} \mathrm{T}$ cells; complete response

\section{Introduction}

Neoadjuvant chemoradiotherapy (NCRT) is indicated in patients with locally advanced rectal cancer (LARC) to downstage tumors before surgery. The current standard of care consists of short or long-course radiotherapy combined with single-agent fluoropyrimidine-based chemotherapy or in combination with oxaliplatin. Following NCRT, a complete clinical response is observed in $10-40 \%$ of patients [1]. Inadequate 
treatment response is associated with an unfavorable long-term prognosis [2]. For patients obtaining a complete clinical response, watchful waiting may be an alternative treatment option allowing certain patients to undergo an organ-preserving strategy and avoid major surgery [3]. The achievement of complete response is affected by the initial clinical UICC stage, pathological subtype, pretherapeutic carcinoembryonic antigen levels, and other currently unidentified factors $[1,4]$. Some studies have identified the density of tumorinfiltrating lymphocytes (TILs) as a predictive marker of treatment response and long-term prognosis in patients with LARC undergoing NCRT [5,6]. Assessment of TILs is not yet included as a part of the routine histopathological examination. However, in research, immunohistochemical (IHC) analysis has been used to assess TILs density and specific subgroups of lymphocytes. $\mathrm{CD}^{+}, \mathrm{CD}^{+}, \mathrm{CD}^{+}$, and $\mathrm{FOXP3}^{+}$TILs are among the most commonly evaluated markers [7]. High levels of $\mathrm{CD}^{+}, \mathrm{CD}^{+}$, and $\mathrm{CD}^{+}$TILs in the tumor microenvironment are reported to be a signature of active antitumor immunity. In contrast, high levels of FOXP3 ${ }^{+}$TILs have been associated with an immunosuppressive tumor microenvironment [8-11]. Infiltration of TILs, especially CD8 ${ }^{+} \mathrm{T}$ cells, in pretherapeutic tumor tissue has been correlated with improved survival in different gastrointestinal cancers, including gastric cancer, hepatocellular carcinoma, and pancreatic cancer [12-14].

This review and meta-analysis examines TILs in pretherapeutic biopsies and their significance in the prediction of treatment response and long-term prognosis in patients with LARC undergoing NCRT and surgical resection.

\section{Materials and Methods}

\subsection{Protocol and Registration}

This study was designed according to the PRISMA-P guidelines [15] for systematic review and meta-analysis. A PRISMA checklist is available in Table S1, Supplementary Material. The protocol was registered at the Center for Open Science under the digital object identifier: osf.io/npgux (accessed on 18 August 2020)

\subsection{Search Strategy}

The literature search was conducted using PubMed, Embase, Cochrane Library, and Web of Science. A Population-Intervention-Comparator-Outcome (PICO)-based search strategy was applied. The population of interest was restricted to Union for International Cancer Control (UICC) stage II-IV human rectal cancers undergoing NCRT before curative intended surgical resection. Assessment of TILs density by at least one of the following IHC markers CD3, CD4, CD8, or FOXP3 in pretherapeutic biopsies was the intervention. As comparators, we considered patients with low versus high TILs density and patients with treatment response versus patients with no response. The outcomes of interest were pathological complete response (pCR) and pathological treatment response (pTR), overall survival (OS), and disease-free survival (DFS) or recurrence-free survival (RFS). pCR and pTR were evaluated histopathologically in the surgical specimen using the tumor regression grade (TRG). There was no restriction regarding the TRG system applied for evaluation.

The search was restricted to human trials and English language articles. Studies with a sample size below 30 were deemed too small to provide relevant information and were excluded. Case studies, reviews, conference abstracts, commentaries, and letters to editors were also excluded. No search restrictions regarding publication date or study design were included in the final search string. The search strategy is summarized with reference to the PICO process in Table S2, Supplemental. The last search was performed on 12 December 2020.

\subsection{Data Management and Extraction}

Search results from the four databases were imported into EndnoteX9 desktop application (Clarivate, Philadelphia, PA, USA) for duplicate removal. After removing duplicates, the articles were imported into Covidence web application (Veritas Health Innovation, Melbourne, Australia) for article screening. Subsequently, two reviewers, FK and AO, inde- 
pendently screened articles at the title and abstract level, followed by full-text screening. The relevance of items was decided based on the predefined eligibility criteria. Any disagreement was resolved by a consensus decision with the inclusion of a third reviewer (RV).

Study data including UICC tumor stage, treatment type, evaluated subgroups of lymphocytes, TILs density, and quantification method (manual vs. digital), as well as the cut-off value for low vs. high densities (median, mean, combined scores, or others), TRG and TRG system were extracted and registered. Corresponding authors of studies with insufficient or missing data were contacted by email, and in case of no reply, studies were excluded from the meta-analysis.

\subsection{Outcomes and Subgroups}

The primary outcome was $\mathrm{pCR}$, defined as no residual cancer cells left in the surgical specimen according to the Dworak, Mandard, and AJCC TRG systems [16-18]. Studies using other TRG systems to assess $\mathrm{pCR}$ were included in the meta-analyses if a pCR did not vary from this definition. Patients not achieving a $\mathrm{pCR}$ were grouped as non-responders and used as comparators in the dichotomous analyses.

The secondary outcome was pTR, defined as a complete or near-complete tumor regression corresponding to a Dworak TRG 3-4, Mandard TRG 1-2, or AJCC TRG 0-1. Studies using other TRG systems were included in the meta-analyses of pTR if the definition did not vary significantly. This outcome was selected as many studies had not differentiated between these two subgroups with no or only minute foci of residual tumor. Patients not achieving pTR were grouped as non-responders and used as comparators in the dichotomous analyses.

OS and DFS/RFS were also included as secondary outcomes reflecting long-term prognosis. The definitions of OS and DFS were based on The National Cancer Institute's Dictionary of Cancer Terms. OS was defined as the time between diagnosis of rectal cancer and the date of death regardless of cause. DFS was defined as the time elapsing from the date of surgery to the first symptoms of recurrent disease or death. We defined RFS as equivalent to DFS, and studies with data on RFS were included in the meta-analysis for DFS. Patients with stage IV disease, and treated with non-curative intent, were excluded from the meta-analyses of survival outcomes.

\subsection{Data Extraction}

Hazard ratios (HR) with corresponding 95\% confidence intervals (CIs) of high vs. low density of TILs were extracted. In studies where only the Kaplan-Meier (KM) plots were available and HR was not presented, the HR was estimated through the KM plots using the DigitizeIt desktop application and methods as described by Tierney et al. [19].

\subsection{Statistical Analysis}

The meta-analyses were conducted separately for each subgroup of TILs and outcomes of interest. All meta-analyses were performed in R statistical software (version 3.4.3, R Foundation for Statistical Computing) applying the Meta [20], Metagen [21], and Metafor [22] packages. Dichotomous outcomes were analyzed using a random-effects model calculating risk ratios (RR) using the Mantel-Haenszel method. Sidik-Jonkman was applied for tau ${ }^{2}$ estimation and Hartung-Knapp adjustment of 95\% CI for the random-effect model [23]. When applicable, prediction intervals supplying an estimate of the expected effect sizes on future studies were calculated [24]. Heterogeneity was assessed by $\mathrm{Chi}^{2}$ testing and $\mathrm{I}^{2}$ statistics. A random-effect model using the inverse variance to estimate HR was applied in meta-analyses of time to event outcomes. The estimate of variance in the meta-analysis was based on upper and lower $95 \%$ CI as described by Altman et al. [25], and analysis was performed as described for dichotomous outcomes. 


\subsection{Risk of Bias of Individual Studies}

The risk of bias within studies was assessed using the Newcastle-Ottawa Scale (NOS) for cohort studies [26]. The NOS examines three domains of a study: patient selection, cohort comparability, and outcome assessment. The maximum attainable NOS score was nine points.

\subsection{Level of Evidence}

The level of evidence was evaluated using a modified GRADE approach for observational studies. The modified version of the GRADE approach can specifically be used in systematic reviews of prognostic markers. According to this approach, the cumulative evidence can be rated as either of high, moderate, low, or very low quality [27]. Each meta-analysis result starts as high-quality evidence. Several limitations can reduce the quality of evidence (risk of bias, inconsistency of results, indirectness, imprecision, and publication bias). Limitations considered not serious do not downgrade evidence. Serious limitations downgrade quality with one level, and very serious limitations downgrade quality with two levels. In contrast, strengths of evidence (large effect size and dose-response gradient) can upgrade the level of evidence. Each strength of evidence upgrades quality with one level.

\section{Results}

\subsection{Search Results}

The literature search identified 429 articles (Figure 1). After removing duplicated articles, a total of 307 studies were screened for title and abstract, ultimately revealing 86 studies for further full-text screening. After the full-text screening was completed, 19 studies were eligible for inclusion in the systematic review [5,6,9-11,28-41]. Of these, 14 studies were eligible $[5,6,9,10,28-37]$ and five studies were ineglible for meta-analysis [11,38-41] (Figure 1). The five studies ineligible in the meta-analysis were due to the following reasons: Three studies were excluded from the meta-analysis as the subgroups of TILs were not dichotomized into high or low-density groups for each individual marker $[38,39,41]$, whereas two were ineligible as the studies included patients that did not undergo curatively intended treatment $[11,40]$.

\subsection{Study Characteristics}

The included studies were conducted in Asia $(n=12)$, Europe $(n=4)$, Middle East $(n=2)$, and Australia $(n=1)$. The studies were published between 2011 and 2020. All studies were retrospective cohort studies. The combined cohort, based on all the eligible studies in the systematic review, consisted of 2034 patients. Most studies included UICC stage II-III cancers, although three studies also included stage IV cancers eligible for curative intended therapy $[6,11,40]$. All patients underwent NCRT before curative intended surgery. Long-course NCRT was used in 16 studies (84\%), whereas two studies did not specify the type of NCRT [5,38], and one study used short-course NCRT [28]. All studies assessed TILs in the diagnostic biopsies using IHC stainings. Different cut-off values were used for dichotomizing the TILs into groups of high vs. low densities. Most studies used the median TILs value, yet mean values, combined scores, and unspecified values were also used. Fifteen studies used Dworak, Mandard, or AJCC for the assessment of TRG. Two studies used the Japanese Colorectal Cancer Classification (JCCC) TRG system [6,30], while two studies did not specify which TRG system they used [31,38] (Table 1). 


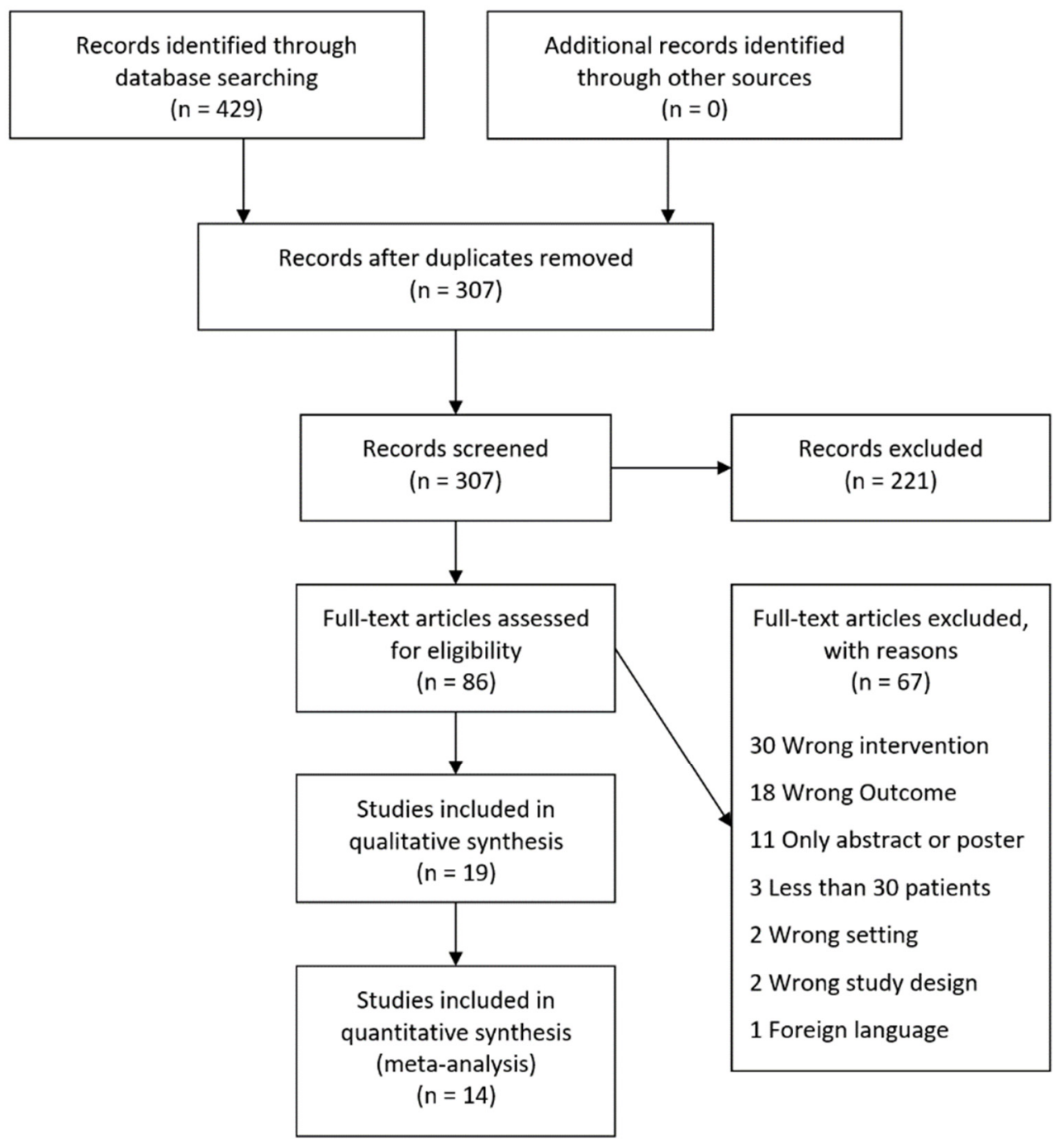

Figure 1. PRISMA flowchart. The literature search was performed using PubMed, Embase, The Cochrane Library, and Web of Science.

Table 1. Main characteristics of the included studies. The density of TILs was assessed in the diagnostic biopsy using IHC with antibodies targeting specific subgroups of TILs. Different cutoff values were used to separate patients into high vs. low density of TILs. Treatment response was evaluated histopathologically using TRG. Treatment response was defined as pCR or pTR in most studies.

\begin{tabular}{cccccc}
\hline Author & UICC Stage & $\begin{array}{c}\text { TILs Density } \\
\text { Cut-Off }\end{array}$ & TRG System & $\begin{array}{c}\text { Treatment Response } \\
\text { Defined as }\end{array}$ & $\begin{array}{c}\text { Inclusion in } \\
\text { Meta-Analysis }\end{array}$ \\
\hline $\begin{array}{c}\text { Anitei et al. [5] } \\
\text { Yasuda et al. [6] }\end{array}$ & II-III & Median & Dworak & TRG 3-4 & CD3 (pTR) \\
II-IV & Unspecified & JCCC & $\begin{array}{c}\text { CD8 (pCR) } \\
\text { CD4 (pTR) }\end{array}$ & FOXP3 (pTR, DFS) \\
Teng et al. (A) [9] & II-III & Median & Dworak & TRG 3-4 & CD8 (pTR, OS, DFS) \\
& & & & CD3 (pTR) \\
Teng et al. (B) [10] & II-III & Median & Dworak & TRG 3-4 & CD8 (pTR, OS, DFS) \\
McCoy et al. [11] & II-IV & Median & Dworak & TRG 3-4 & none \\
Shinto et al. (A) [28] & II-III & Median & Dworak & TRG 3-4 & FOXP3 (pTR, DFS) \\
& & & & &
\end{tabular}


Table 1. Cont.

\begin{tabular}{|c|c|c|c|c|c|}
\hline Author & UICC Stage & $\begin{array}{l}\text { TILs Density } \\
\text { Cut-Off }\end{array}$ & TRG System & $\begin{array}{c}\text { Treatment Response } \\
\text { Defined as }\end{array}$ & $\begin{array}{l}\text { Inclusion in } \\
\text { Meta-Analysis }\end{array}$ \\
\hline Shinto et al. (B) [29] & II-III & Unspecified & Dworak & pCR, TRG 3-4 & $\begin{array}{c}\text { CD8 (pCR, pTR) } \\
\text { CD4 (pTR) }\end{array}$ \\
\hline Matsutani et al. [30] & II-III & Median & JCCC & TRG $1 b-3$ & $\begin{array}{c}\text { FOXP3 (pTR) } \\
\text { CD8 (pTR) }\end{array}$ \\
\hline Zaghloul et al. [31] & II-III & ROC & Unspecified & $\mathrm{pCR}$ & $\begin{array}{c}\text { FOXP3 (DFS) } \\
\text { CD8 (pCR, OS, DFS) } \\
\text { CD4 (pTR) }\end{array}$ \\
\hline Zhang et al. [32] & II-III & Mean & Dworak & TRG 3-4 & $\begin{array}{c}\text { FOXP3 (pTR) } \\
\text { CD8 (pTR) }\end{array}$ \\
\hline Akiyoshi et al. [33] & II-III & Median & Dworak & pCR, TRG 3-4 & $\begin{array}{c}\text { CD8 (pCR, pTR, } \\
\text { DFS) }\end{array}$ \\
\hline Chen et al. [34] & II-III & Unspecified & Dworak & TRG 3-4 & CD8 (OS, DFS) \\
\hline Moghani et al. [35] & II-III & $\begin{array}{l}11 \text { cells /high } \\
\text { power field }\end{array}$ & AJCC & pCR, TRG 0-1 & CD8 (pCR, pTR) \\
\hline Xiao et al. [36] & II-III & Unspecified & Mandard & $\mathrm{pCR}$ & CD8 (pCR) \\
\hline Huang, Y et al. [37] & II-III & Unspecified & AJCC & TRG 0-1 & $\begin{array}{l}\text { CD4 (pTR) } \\
\text { CD8 (pTR) }\end{array}$ \\
\hline Mirjolet et al. [38] & II-III & None & Unspecified & Unspecified & none \\
\hline Huang, A et al. [39] & II-III & $\begin{array}{l}\text { CD3/CD8 } \\
\text { combined }\end{array}$ & Dworak & TRG 3-4 & none \\
\hline Rudolf et al. [40] & II-IV & Median & Dworak & Unspecified & none \\
\hline Sissy et al. [41] & II-III & $\begin{array}{l}\text { CD3/CD8 } \\
\text { combined }\end{array}$ & Dworak & Unspecified & none \\
\hline
\end{tabular}

UICC: Union for International Cancer Control. AJCC: American Joint Committee on Cancer. JCCC: Japanese Colorectal Cancer Classification. TRG: Tumor regression grade. pCR: Pathological complete response. pTR: Pathological treatment response.

\subsection{Pathological Complete Response ( $p C R$ )}

Six studies were eligible for the meta-analysis of the primary outcome, pCR. In four studies, Dworak, Mandard, or AJCC [29,33,35,36] were used, while two studies used other TRG systems [6,31]. The eligible studies provided information regarding high versus low density of CD8 ${ }^{+}$TILs on 692 patients in total. Of the patients included in the meta-analysis, 282 patients $(41 \%)$ were categorized as having a high CD8 ${ }^{+}$TILs density and 410 patients $(59 \%)$ with having a low $\mathrm{CD} 8^{+}$TILs density. The categorization of patients in either the high or low $\mathrm{CD} 8^{+} \mathrm{T}$ cell expression groups was based on different cut-off estimation methods in the six studies included in the meta-analysis. Three studies did not specify the cut-off estimation $[6,29,36]$, one of the studies based the cut-off on the precalculated median TIL value in the cohort [33], whereas one used 11 cells per high power field as the cut-off value [35], and the last study used a receiver operating characteristics (ROC) curve [31] (Table 1). Patients with high CD8 ${ }^{+}$TILs density had an increased likelihood of achieving a pCR compared to patients with a low $\mathrm{CD} 8^{+}$TILs density (RR $=2.71 ; 95 \% \mathrm{CI}$ : $\left.1.58-4.66\right)$ (Figure 2).

Meta-analysis on the association between pretherapeutic $\mathrm{CD}^{+}, \mathrm{CD}^{+}$, and $\mathrm{FOXP}^{+}$TILs and PCR could not be performed due to a lack of studies reporting data on these variables. 


\begin{tabular}{lrrrr} 
& \multicolumn{2}{c}{$\begin{array}{c}\text { High CD8 } \\
\text { Events }\end{array}$} & \multicolumn{2}{c}{ Low CD8 } \\
& 9 & 23 & 15 & 69 \\
Xiao & 7 & 24 & 1 & 24 \\
Yasuda & 6 & 28 & 2 & 22 \\
Zaghloul & 29 & 138 & 14 & 137 \\
Akiyoshi & 8 & 41 & 3 & 103 \\
Shinto (B) & 10 & 28 & 6 & 55 \\
Moghani & & & & \\
& & 282 & & 410 \\
Random effects model & & & & \\
Prediction interval & & & & \\
Heterogeneity: $I^{2}=0 \%, \tau^{2}=0.1461, p=0.42$ & &
\end{tabular}

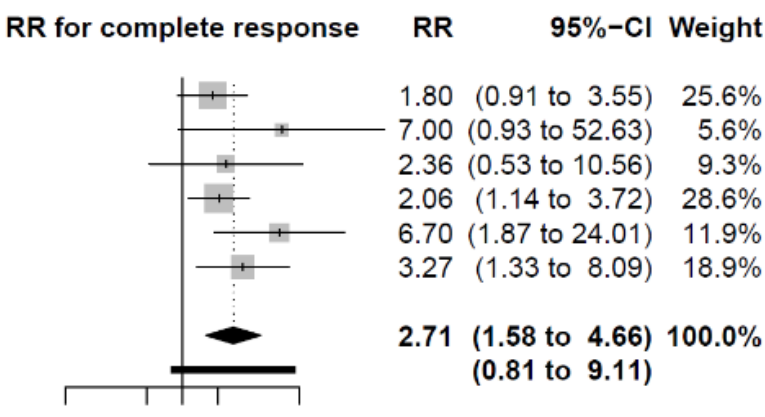

$0.1 \quad 0.512 \quad 10$

Figure 2. The relationship between $\mathrm{CD} 8^{+}$TILs density and $\mathrm{PCR}$. Patients with high pretherapeutic $\mathrm{CD}^{+}$TILs density had a significantly increased likelihood of achieving a pCR. RR: Risk Ratio.

\subsection{Pathological Treatment Response ( $p$ TR)}

Overall, 10 studies were eligible for the meta-analyses of the secondary outcome, pTR. Nine studies assessed TRG according to Dworak, Mandard, or AJCC [5,9,10,28,29,32,33,35,37]. Matsutani et al. used JCCC and included TRG 1b-3 in the group of pTR [30].

\subsection{1. $\mathrm{CD}^{+} \mathrm{TILs}$}

Nine studies providing data on 1073 patients in total were included in the metaanalysis on pretherapeutic CD8 ${ }^{+}$TILs density and pTR [9,10,28-30,32,33,35,37]. In total, 491 patients $(46 \%)$ were categorized with high CD8 ${ }^{+}$TILs levels, while $582(54 \%)$ of the patients were categorized with low $\mathrm{CD} 8^{+}$TILs levels. Again, the differentiation between high versus low levels of the examined lymphocyte subsets was primarily based on a precalculated median value of the examined cohort in each of the eligible studies. Patients with high levels of $\mathrm{CD} 8^{+}$TILs had an increased likelihood of obtaining a pTR (RR $=1.86$; 95\% CI: 1.50-2.29) (Figure 3).

High CD8 Low CD8

Study

Shinto $(A)$

Teng (A)

Teng (B)

Zhang

Huang

Akiyoshi

Matsutani

Shinto (B)

Moghani

\section{Events Total Events Total}

$\begin{array}{rrrr}22 & 48 & 11 & 45 \\ 19 & 31 & 10 & 31 \\ 42 & 75 & 22 & 61 \\ 18 & 46 & 16 & 63 \\ 35 & 69 & 26 & 72 \\ 45 & 138 & 23 & 137 \\ 12 & 15 & 2 & 15 \\ 27 & 41 & 29 & 103 \\ 19 & 28 & 19 & 55 \\ & & & \\ & \mathbf{4 9 1} & & \mathbf{5 8 2}\end{array}$

Prediction interval

Heterogeneity: $I^{2}=0 \%, \tau^{2}=0.0661, p=0.45$
RR for response

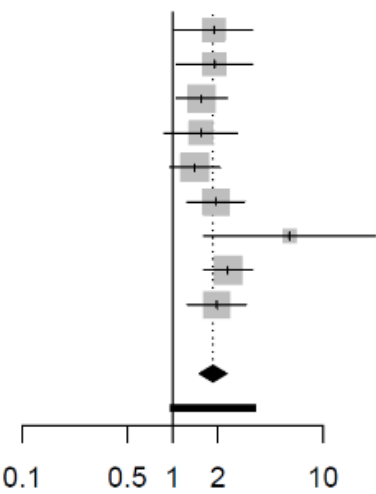

RR $\quad 95 \%-\mathrm{Cl}$ Weight

1.88 (1.03 to 3.41$) \quad 9.3 \%$

1.90 (1.06 to 3.40$) \quad 9.6 \%$

1.55 (1.05 to 2.29 ) $14.1 \%$

1.54 (0.88 to 2.69 ) $10.1 \%$

1.40 (0.96 to 2.07 ) $14.2 \%$

1.94 (1.25 to 3.03 ) $12.7 \%$

6.00 (1.61 to 22.34 ) $\quad 2.9 \%$

2.34 (1.60 to 3.42 ) $14.4 \%$

1.96 (1.26 to 3.06 ) $12.7 \%$

$1.86(1.50$ to 2.29$) 100.0 \%$

(0.97 to 3.54 )

Figure 3. The relationship between CD8 ${ }^{+}$TILs density and pTR. Patients with high CD8 ${ }^{+}$TILs density had a significantly increased likelihood of achieving a pTR. RR: Risk Ratio.

\subsection{2. $\mathrm{CD}^{+}$TILs}

Two studies with 191 patients in total investigated the association between pretherapeutic $\mathrm{CD}^{+}$TILs density and pTR $[5,10]$. Of the total, $102(53 \%)$ patients were categorized as $\mathrm{CD}^{+}$TILs high, whereas the remaining $89(47 \%)$ patients had low $\mathrm{CD}^{+}$lymphocyte infiltration. High density of $\mathrm{CD}^{+}$TILs was not significantly associated with any changes in pTR (RR $=1.63$; 95\% CI: 0.35-7.69, $p=0.16)$ (Figure S1, Supplemental). 


\subsection{3. $\mathrm{CD}^{+}$TILs}

Four studies consisting of 342 patients altogether, evaluated the association between pretherapeutic CD4 ${ }^{+}$TILs density and pTR [9,30,32,37]. High CD4 ${ }^{+}$TILs were reported in 175 patients (51\%), while the remaining 167 (49\%) of patients in the total cohort had low expression of $\mathrm{CD}^{+}{ }^{+}$TILs. CD4 ${ }^{+}$TILs density was not correlated with significant changes in pTR $(R R=1.23 ; 95 \%$ CI: 0.83-1.82, $p=0.19)$ (Figure S2, Supplemental).

\subsubsection{FOXP3 ${ }^{+}$TILs}

Four studies with 294 patients in total examined the association between pretherapeutic FOXP3 ${ }^{+}$TILs density and pTR [9,28,30,32]. Overall, 129 patients (44\%) were categorized as having high $\mathrm{FOXP3}^{+}$TILs expression, while the remaining $165(56 \%)$ had low expression. The meta-analysis revealed no significant association between FOXP3 ${ }^{+}$TILs density and pTR $(\mathrm{RR}=0.85 ; 95 \%$ CI: 0.20-3.58, $p=0.74)$ (Figure S3, Supplemental).

\subsection{Overall Survival (OS)}

Four studies providing data on 360 patients altogether examined the association between $\mathrm{CD}^{+}$TILs and OS $[9,10,31,34]$. Of the total number of patients, $170(47 \%)$ patients had high $\mathrm{CD}^{+}$lymphocyte infiltration and $190(53 \%)$ patients had low $\mathrm{CD}^{+}$lymphocyte infiltration. Meta-analysis of time-to-event data revealed that a high CD8 ${ }^{+}$TILs density was a significantly favorable prognostic factor for OS (HR $=0.43$; 95\% CI: 0.27-0.69) (Figure 4).

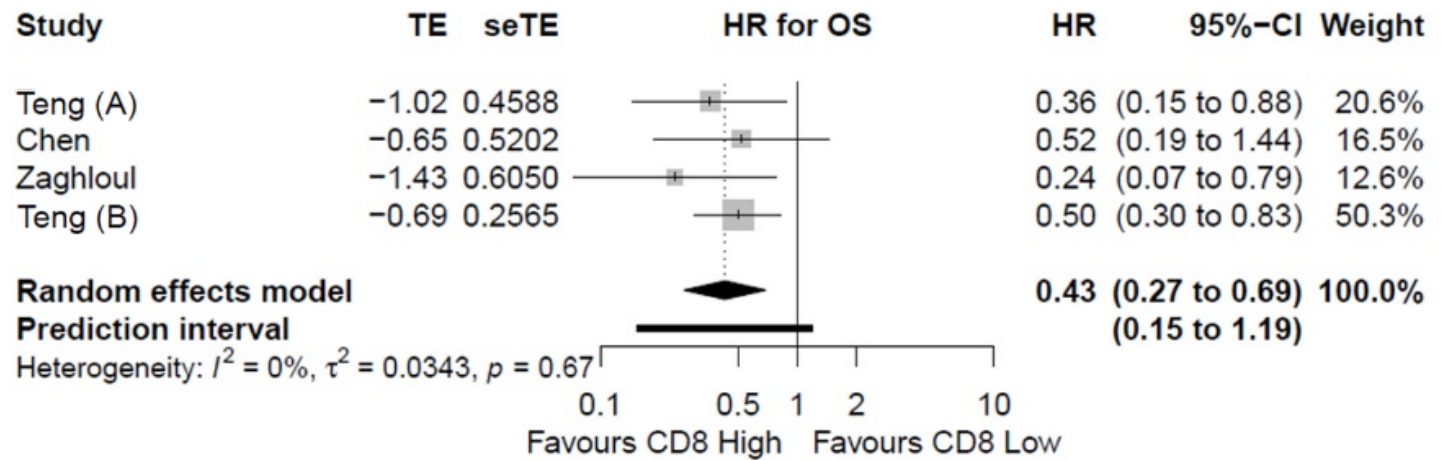

HR for DFS

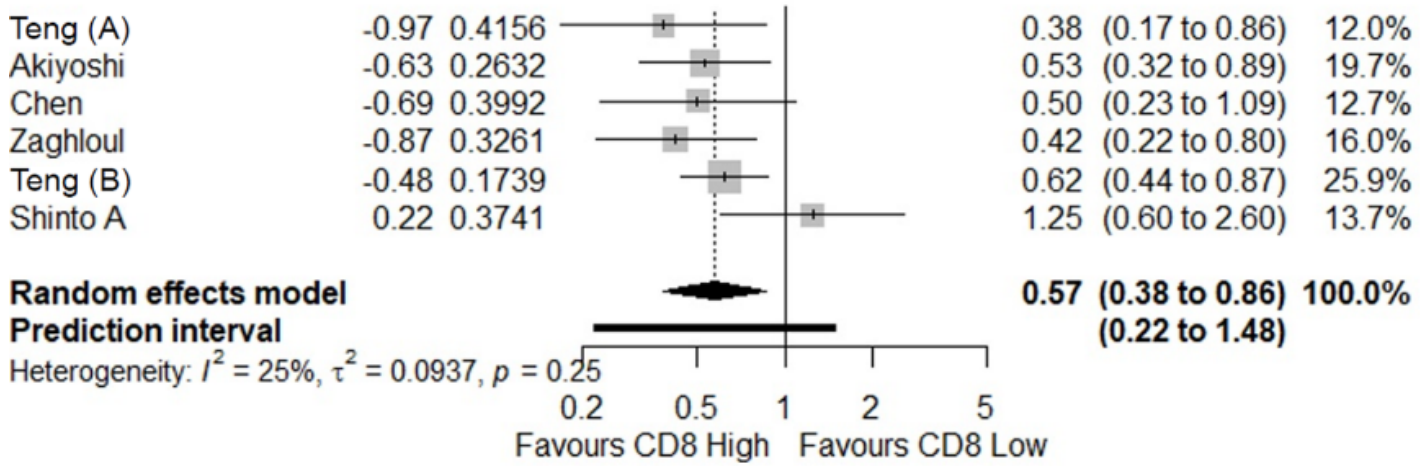

Figure 4. The prognostic value of $\mathrm{CD}^{+}$TILs. Meta-analyses of time-to-event data indicated a favorable overall survival (OS) and disease-free survival (DFS) for patients with high pretherapeutic $\mathrm{CD}^{+}$TILs density. HR: Hazard Ratio.

\subsection{Disease-Free Survival (DFS)}

\subsection{1. $\mathrm{CD}^{+} \mathrm{TILs}$}

Six studies consisting of 716 patients overall evaluated the association between CD8 ${ }^{+}$ TILs levels and DFS [9,10,28,31,33,34]. In sum, $349(49 \%)$ and $367(51 \%)$ of the patients were 
categorized with high and low $\mathrm{CD}^{+}$TILs density, respectively. Time-to-event analysis revealed that a high $\mathrm{CD}^{+}$TILs density was significantly associated with improved DFS $(\mathrm{HR}=0.57 ; 95 \% \mathrm{CI}: 0.38-0.86)$ (Figure 4$)$.

\subsection{2. $\mathrm{FOXP3}^{+} \mathrm{TILs}$}

Three studies with a sum of 193 patients examined whether the pretherapeutic FOXP3 ${ }^{+}$ TILs level was a prognostic factor for DFS [9,28,31]. Collectively, 98 (51\%) of the patients were categorized as having a high FOXP3 ${ }^{+}$TILs level, while 95 (49\%) of the patients were categorized as having a low FOXP3 ${ }^{+}$TILs level. The FOXP3 ${ }^{+}$TILs density was not associated with any significant changes in DFS (HR $=1.66$; 95\% CI: 0.17-16.32) (Figure S4, Supplemental).

\subsection{Risk of Bias of Individual Studies}

Using the NOS scoring system, four of the included studies in the systematic review achieved seven points out of a maximum of nine points $[5,31,35,40]$. Ten studies achieved eight points $[6,9-11,29,30,32,33,36,38]$. Five studies achieved the maximum NOS score of nine points $[28,34,37,39,41]$.

Ten studies were downgraded in the comparability section as the studies did not control for confounders $[5,9-11,30-32,35,38,40]$. Seven studies were downgraded in the outcome section as the studies did not report whether TILs were assessed by independent and blinded assessors [5,29,31,33,35,36,40]. A detailed description is available in Table S3, Supplementary Material.

\subsection{Level of Evidence}

Using the GRADE approach, the level of evidence was rated as moderate on all outcomes for CD8 ${ }^{+}$TILs. For pCR and OS, the quality of the cumulative evidence was downgraded two levels due to potential publication bias and imprecision but upgraded one level due to a large effect size. For pTR and DFS, the quality of evidence was downgraded one level due to potential publication bias. The quality of the cumulative evidence for the results related to the $\mathrm{CD}^{+}, \mathrm{CD}^{+}$, and $\mathrm{FOXP3}^{+}$TILs subsets was rated as low or very low due to multiple limitations. Publication bias could not be statistically assessed by funnel plots due to the limited number of studies, and therefore, all results were downgraded one level. A detailed overview is available in Tables S4 and S5, Supplementary Material.

Table 2 provides an overview of the abovementioned results, including the quality of the cumulative evidence based on the modified GRADE approach.

Table 2. This table provides an overview of the results of the meta-analyses including a point estimate of the risk ratio (in relation to pathological response) and the hazard ratio (in relation to survival) with corresponding CI. The level of evidence has also been included in the table. The meta-analyses evaluated the association between pretherapeutic TILs density and treatment response to NCRT and long-term prognosis.

\begin{tabular}{|c|c|c|c|c|c|c|c|c|}
\hline Outcome & Biomarker & Studies & $n$ & Point Estimate & Lower $95 \%$ CI & Higher $95 \%$ CI & $I^{2}$ & $\begin{array}{l}\text { GRADE Level } \\
\text { of Evidence }\end{array}$ \\
\hline \multirow[t]{2}{*}{$\mathrm{pCR}$} & $\mathrm{CD}^{+}{ }^{+} \mathrm{TILs}$ & 6 & 692 & 2.71 & 1.58 & 4.66 & $0 \%$ & Moderate \\
\hline & $\mathrm{CD}^{+}$TILs & 2 & 191 & 1.63 & 0.35 & 7.69 & $0 \%$ & Low \\
\hline \multirow{3}{*}{ pTR } & $\mathrm{CD}^{+}{ }^{+}$TILs & 4 & 342 & 1.23 & 0.83 & 1.82 & $0 \%$ & Low \\
\hline & CD8 ${ }^{+}$TILs & 9 & 1073 & 1.86 & 1.50 & 2.29 & $0 \%$ & Moderate \\
\hline & $\mathrm{FOXP3}^{+}$TILs & 4 & 294 & 0.85 & 0.20 & 3.58 & $76 \%$ & Very low \\
\hline \multirow{2}{*}{ DFS } & CD8 ${ }^{+}$TILs & 6 & 716 & 0.57 & 0.38 & 0.86 & $25 \%$ & Moderate \\
\hline & $\mathrm{FOXP3}^{+}$TILs & 3 & 193 & 1.66 & 0.17 & 16.32 & $62 \%$ & Very low \\
\hline OS & CD8 ${ }^{+}$TILs & 4 & 360 & 0.43 & 0.27 & 0.69 & $0 \%$ & Moderate \\
\hline
\end{tabular}

pCR: Pathological complete response. pTR: Pathological treatment response. DFS: Disease-free survival. OS: Overall survival. $n$ : number of patients. CI: Confidence interval. 


\section{Discussion}

In this systematic review and meta-analysis, we investigated the pretherapeutic TILs density as a predictive marker of treatment response and long-term prognosis in patients with LARC undergoing NCRT and surgery. The meta-analysis found that patients with a high pretherapeutic $\mathrm{CD} 8^{+}$TILs density had an increased likelihood of achieving $\mathrm{pCR}$ and pTR. Correspondingly, patients with a high pretherapeutic CD8 ${ }^{+}$TILs density had a favorable DFS and OS compared to patients with low CD8 ${ }^{+}$TILs infiltration. Our findings are consistent with previously published meta-analyses that have correlated high $\mathrm{CD} 8^{+}$ TILs density to a favorable prognosis for patients with colorectal cancer $[7,8]$.

It is hypothesized that patients with rectal cancer having a high pretherapeutic TILs density are more likely to have a good response to NCRT, as the preexisting antitumor immunity is enhanced by NCRT. This hypothesis is supported by preclinical studies showing that CRT alters the tumor microenvironment (TME) in favor of antitumor immunity $[42,43]$. Tumor cell death following CRT causes a release of tumor neoantigens and pro-inflammatory mediators into the TME, increasing T-cell infiltration, T-cell elimination of cancer cells, and facilitating further adaptive antitumor responses [44-47].

Combining NCRT with immunotherapy may additionally enhance the T-cell mediated antitumor response [48-50]. Multiple clinical trials are currently investigating whether the addition of immunotherapy to NCRT regimens increases $\mathrm{pCR}$ rates in patients with LARC (ClinicalTrials.gov (accessed on 6 January 2022): NCT04017455, NCT03127007, NCT03102047, NCT03854799, and NCT03921684). Recently, a phase II randomized clinical trial examined the efficacy and safety of adding the immune check-point inhibitor (ICI), pembrolizumab, to standard NCRT for the treatment of rectal cancer [51]. The study found $\mathrm{pCR}$ in $31.9 \%$ and $29.4 \%$ of the patients in the pembrolizumab arm and control arm, respectively. The findings did not reach statistical significance, and the percentage of grade 3 and 4 adverse events were slightly higher in the pembrolizumab arm [51]. However, the study did not examine the pretherapeutic TILs level, the amount of tumor stroma, the number of cancer-associated fibroblasts (CAFs), or other TME factors associated with a tumor phenotype that negatively impacts response rates and survival measures $[52,53]$. The composition of the TME highly influences the infiltration, survival, and proliferation of TILs. Fibrotic tissue surrounding cancer cells may prevent direct cell-to-cell contact between lymphocytes and cancer cells, thus hindering the killing mechanism of malignant cells by lymphocytes. Also, hypoxia in the TME may lead to apoptosis of TILs, thereby reducing their number and effectivity in the tumor tissue [53].

Examining the density of TILs as well as the composition of the TME prior to treatment with ICI have the potential to further assist the clinicians in the selection of patients that may benefit from this treatment. However, tumor sampling by using single biopsies has been associated with limitations [54]. The tumoral immune infiltrate and the composition of the TME may be better evaluated with multiple biopsies or by selecting a larger tumor area for sampling [55]. These considerations should also be taken into account when analyzing and interpreting the TILs density and the overall composition of the TME. In continuation, the Immunoscore has been internationally validated as a prognostic tool for colon cancer [56,57]. The Immunoscore is a scoring system categorizing patients as low, intermediate, or high based on the density of $\mathrm{CD} 8^{+}$and $\mathrm{CD}^{+} \mathrm{T}$ cells and their abundance in the center and margin of the tumor.

The international validation studies on the Immunoscore in colon cancer have found the method to be a reliable estimate of recurrence in patients with colon cancer with superiority to several routinely assessed parameters as histopathological differentiation, microsatellite instability (MSI) status, and vascular and perineural invasion [56,57]. Likewise, a diagnostic biopsy-adapted immunoscore has been found to be a predictive method for both survival and neoadjuvant treatment response in patients with LARC undergoing surgery [41]. This approach may also assist in selecting patients with LARC that may benefit from a "watchful waiting" regimen when planning the treatment in the oncological and surgical setting. 
Limitations of this study should also be addressed. Firstly, the GRADE assessment revealed low to moderate levels of evidence. For many of the examined outcomes and TILs subgroups examined in this study, the number of data eligible for the meta-analysis was limited and the results for some of the outcomes and TILs subgroups were thus based on small sample sizes. The limited number of studies also prevented proper assessment of the risk of publication bias. Secondly, our evaluation of the risk of bias in individual studies revealed several limitations. All the included studies were retrospective cohort studies creating a risk of selection or information bias. Some of the included studies did not clarify if experienced and blinded pathologists assessed the TRG and TILs density. Evaluation of TRG is associated with significant interobserver variability [58], and only a few of the included studies have addressed this problem. Our meta-analyses combined TRG assessment using various TRG systems. Although the TRG systems are quite similar, minor differences exist with the risk of increasing the imprecision of the results. Furthermore, most studies assessed TILs density manually and not digitally. The manual assessment of TILs is associated with interobserver variability, which may result in a considerate inconsistence in the reported data. Complete pathological response is usually not associated with interobserver variability. However, most pathologists find tumor regression grading difficult. Furthermore, the histological examination including number of examined sections from each tumor block is not uniform among pathologists [59]. In the United Kingdom, it is required to examine additional deeper sections from each block before reporting a diagnosis of pCR [60], while this is not mentioned as obligatory in the recommendations by the International Collaboration of Cancer Reporting (ICCR) [61].

Likewise, different cut-off values were used to classify the patients into either high or low TILs density groups in the included studies, creating an inconsistence in the cumulative evidence. Most of the included studies used the precalculated median value of the study cohort as the cut-off value to classify patients into either high or low lymphocyte infiltration groups. The median value of TILs may vary, not just across different cancer types, but also in different cohorts of patients with the same cancer. This variability in the categorization of patients should be taken into account when interpreting the results of this meta-analysis. Standardizing the cut-off value of TILs for different cancer types may thus assist in creating a more consistent classification of tumors as having high or low TILs levels. Consensus concerning a standardized cut-off value optimized to predict outcomes as well as an exact definition of the area where to perform the counts would probably result in a more homogenous group and studies would be easier to compare, especially when conducting meta-analyses.

Lastly, our meta-analyses were performed on dichotomized data. While dichotomization of values simplifies statistical analysis and clinical application, it hinders the possibility to evaluate a dose-response relationship between the degree of immune infiltration and the degree of tumor regression. Proving a dose-response relationship would, in theory, strengthen the validity of the findings as outlined in the GRADE approach.

Despite the limitations, the findings of this meta-analysis indicate that assessment of pretherapeutic TILs may identify a subgroup of patients with LARC that may benefit from NRCT with improved treatment response and a favorable long-term prognosis as well as patients that may benefit most from a watchful waiting regimen. Thus, assessment of $\mathrm{CD}^{+}$TILs at time of diagnosis may, among other clinical-biological markers and clinical decision support tools, assist clinicians in selecting patients who may benefit from NCRT and immune-enhancing treatment regimens, establishing a more personalized approach to the treatment of LARC. Further clinical studies examining the association between preand post-therapeutic TILs density and improved prognosis, as well as the survival benefit of adding ICIs to standard neoadjuvant therapy for LARC should be conducted.

\section{Conclusions}

High pretherapeutic CD8 ${ }^{+}$TIL levels were associated with pCR and pTR as well as improved DFS and OS in patients with LARC undergoing NCRT, suggesting the density 
of $\mathrm{CD}^{+}$TILs in the pretherapeutic biopsies may serve as an important predictive and prognostic biomarker of treatment response and survival.

The same results could not be obtained for $\mathrm{CD}^{+}$or $\mathrm{CD} 4^{+}$TILs. Likewise, the findings related to FOXP3 ${ }^{+}$TILs did not reveal any significant results. However, the results on these TILs subtypes were based on limited data. Further examination of the predictive and prognostic value of pretherapeutic TIL levels in rectal cancer is thus needed. Especially, prospective studies using reproducible digital and consistent quantification methods of both TILs and TRG are highly relevant when conducting future studies.

Supplementary Materials: The following supporting information can be downloaded at: https:/ / www.mdpi.com/article/10.3390/cancers14030636/s1, Table S1: PRISMA checklist, Table S2: Search strategy, Table S3: Newcastle-Ottawa Scale, Table S4: GRADE assessment of the quality of evidence for pCR and pTR, Table S5: GRADE assessment of the quality of evidence for DFS and OS, Figure S1: $\mathrm{CD}^{+}$TILs density and pTR, Figure S2: $\mathrm{CD}^{+}$TILs density and pTR, Figure S3: FOXP3 ${ }^{+}$TILs density and pTR, Figure S4: Prognostic value of FOXP3 ${ }^{+}$TIL

Author Contributions: Conceptualization, F.K., A.O., R.P.V. and I.G.; methodology, F.K., A.O., R.P.V., A.-M.K.F. and I.G.; software, M.T.M.; validation, F.K., M.T.M. and R.P.V.; formal analysis, M.T.M., F.K., R.P.V.; investigation, F.K., A.O. and R.P.V.; data curation, F.K. and A.O; writing-original draft preparation, F.K. and A.O.; writing-review and editing, F.K., A.O., M.T.M., R.P.V., N.D., A.-M.K.F. and I.G.; visualization, F.K. and M.T.M.; supervision, I.G. All authors have read and agreed to the published version of the manuscript.

Funding: This research received no external funding.

Conflicts of Interest: The authors declare no conflict of interest.

\section{References}

1. Glynne-Jones, R.; Wyrwicz, L.; Tiret, E.; Brown, G.; Rödel, C.; Cervantes, A.; Arnold, D. Rectal Cancer: ESMO Clinical Practice Guidelines for Diagnosis, Treatment and Follow-Up. Ann. Oncol. 2017, 28, iv22-iv40. [CrossRef] [PubMed]

2. Fokas, E.; Liersch, T.; Fietkau, R.; Hohenberger, W.; Beissbarth, T.; Hess, C.; Becker, H.; Ghadimi, M.; Mrak, K.; Merkel, S.; et al. Tumor Regression Grading After Preoperative Chemoradiotherapy for Locally Advanced Rectal Carcinoma Revisited: Updated Results of the CAO/ARO/AIO-94 Trial. J. Clin. Oncol. 2014, 32, 1554-1562. [CrossRef] [PubMed]

3. Valk, M.J.M.; van der Hilling, D.E.; Bastiaannet, E.; Kranenbarg, E.M.-K.; Beets, G.L.; Figueiredo, N.L.; Habr-Gama, A.; Perez, R.O.; Renehan, A.G.; van de Velde, C.J.H.; et al. Long-Term Outcomes of Clinical Complete Responders after Neoadjuvant Treatment for Rectal Cancer in the International Watch \& Wait Database (IWWD): An International Multicentre Registry Study. Lancet 2018, 391, 2537-2545. [CrossRef] [PubMed]

4. Moureau-Zabotto, L.; Farnault, B.; de Chaisemartin, C.; Esterni, B.; Lelong, B.; Viret, F.; Giovannini, M.; Monges, G.; Delpero, J.-R.; Bories, E.; et al. Predictive Factors of Tumor Response After Neoadjuvant Chemoradiation for Locally Advanced Rectal Cancer. Int. J. Radiat. Oncol. Biol. Phys. 2011, 80, 483-491. [CrossRef]

5. Anitei, M.-G.; Zeitoun, G.; Mlecnik, B.; Marliot, F.; Haicheur, N.; Todosi, A.-M.; Kirilovsky, A.; Lagorce, C.; Bindea, G.; Ferariu, D.; et al. Prognostic and Predictive Values of the Immunoscore in Patients with Rectal Cancer. Clin. Cancer Res. 2014, 20, 1891-1899. [CrossRef]

6. Yasuda, K.; Nirei, T.; Sunami, E.; Nagawa, H.; Kitayama, J. Density of CD4(+) and CD8(+) T Lymphocytes in Biopsy Samples Can Be a Predictor of Pathological Response to Chemoradiotherapy (CRT) for Rectal Cancer. Radiat. Oncol. 2011, 6, 1-6. [CrossRef]

7. Idos, G.E.; Kwok, J.; Bonthala, N.; Kysh, L.; Gruber, S.B.; Qu, C. The Prognostic Implications of Tumor Infiltrating Lymphocytes in Colorectal Cancer: A Systematic Review and Meta-Analysis. Sci. Rep. 2020, 10, 1-14. [CrossRef]

8. Gooden, M.J.M.; de Bock, G.H.; Leffers, N.; Daemen, T.; Nijman, H.W. The Prognostic Influence of Tumour-Infiltrating Lymphocytes in Cancer: A Systematic Review with Meta-Analysis. Br. J. Cancer 2011, 105, 93-103. [CrossRef]

9. Teng, F.; Meng, X.; Kong, L.; Mu, D.; Zhu, H.; Liu, S.; Zhang, J.; Yu, J. Tumor-Infiltrating Lymphocytes, Forkhead Box P3, Programmed Death Ligand-1, and Cytotoxic T Lymphocyte-Associated Antigen-4 Expressions before and after Neoadjuvant Chemoradiation in Rectal Cancer. Transl. Res. 2015, 166, 721-732.e1. [CrossRef]

10. Teng, F.; Mu, D.; Meng, X.; Kong, L.; Zhu, H.; Liu, S.; Zhang, J.; Yu, J. Tumor Infiltrating Lymphocytes (TILs) before and after Neoadjuvant Chemoradiotherapy and Its Clinical Utility for Rectal Cancer. Am. J. Cancer Res. 2015, 5, 2064.

11. McCoy, M.J.; Hemmings, C.; Anyaegbu, C.C.; Austin, S.J.; Lee-Pullen, T.F.; Miller, T.J.; Bulsara, M.K.; Zeps, N.; Nowak, A.K.; Lake, R.A.; et al. Tumour-Infiltrating Regulatory T Cell Density before Neoadjuvant Chemoradiotherapy for Rectal Cancer Does Not Predict Treatment Response. Oncotarget 2017, 8, 19803-19813. [CrossRef]

12. Ding, W.; Xu, X.; Qian, Y.; Xue, W.; Wang, Y.; Du, J.; Jin, L.; Tan, Y. Prognostic Value of Tumor-Infiltrating Lymphocytes in Hepatocellular Carcinoma A Meta-Analysis. Medicine 2018, 97, e13301. [CrossRef] [PubMed] 
13. Lee, J.S.; Won, H.S.; Sun, D.S.; Hong, J.H.; Ko, Y.H. Prognostic Role of Tumor-Infiltrating Lymphocytes in Gastric Cancer A Systematic Review and Meta-Analysis. Medicine 2018, 97, e11769. [CrossRef] [PubMed]

14. Orhan, A.; Vogelsang, R.P.; Andersen, M.B.; Madsen, M.T.; Hölmich, E.R.; Raskov, H.; Gögenur, I. The Prognostic Value of Tumour-Infiltrating Lymphocytes in Pancreatic Cancer: A Systematic Review and Meta-Analysis. Eur. J. Cancer 2020, 132, 71-84. [CrossRef] [PubMed]

15. Moher, D.; Shamseer, L.; Clarke, M.; Ghersi, D.; Liberati, A.; Petticrew, M.; Shekelle, P.; Stewart, L.A. Preferred Reporting Items for Systematic Review and Meta-Analysis Protocols (PRISMA-P) 2015 Statement. Syst. Rev. 2015, 4, 1-9. [CrossRef]

16. Dworak, O.; Keilholz, L.; Hoffmann, A. Pathological Features of Rectal Cancer after Preoperative Radiochemotherapy. Int. J. Colorectal Dis. 1997, 12, 19-23. [CrossRef]

17. Mandard, A.-M.; Dalibard, F.; Mandard, J.-C.; Marnay, J.; Henry-Amar, M.; Petiot, J.-F.; Roussel, A.; Jacob, J.-H.; Segol, P.; Samama, G.; et al. Pathologic Assessment of Tumor Regression after Preoperative Chemoradiotherapy of Esophageal Carcinoma. Clinicopathologic Correlations. Cancer 1994, 73, 2680-2686. [CrossRef]

18. Edge, S.B.; Compton, C.C. The American Joint Committee on Cancer: The 7th Edition of the AJCC Cancer Staging Manual and the Future of TNM. Ann. Surg. Oncol. 2010, 17, 1471-1474. [CrossRef]

19. Tierney, J.F.; Stewart, L.A.; Ghersi, D.; Burdett, S.; Sydes, M.R. Practical Methods for Incorporating Summary Time-to-Event Data into Meta-Analysis. Trials 2007, 8, 1-16. [CrossRef]

20. Balduzzi, S.; Rücker, G.; Schwarzer, G. Statistics in Practice How to Perform a Meta-Analysis with R: A Practical Tutorial. Evid. Based Ment. Health 2019, 22, 153-160. [CrossRef]

21. Möbius, T.W.D. Metagen: Inference in Meta Analysis and Meta Regression. Available online: http://00tau.github.io/metagen/ (accessed on 12 December 2020).

22. Viechtbauer, W. Conducting Meta-Analyses in R with the Metafor Package. J. Stat. Softw. 2010, 36, 1-48. [CrossRef]

23. IntHout, J.; Ioannidis, J.P.; Borm, G.F. The Hartung-Knapp-Sidik-Jonkman Method for Random Effects Meta-Analysis Is Straightforward and Considerably Outperforms the Standard DerSimonian-Laird Method. BMC Med. Res. Methodol. 2014, 14, 1-12. [CrossRef]

24. IntHout, J.; Ioannidis, J.P.A.; Rovers, M.M.; Goeman, J.J. Plea for Routinely Presenting Prediction Intervals in Meta-Analysis. BMJ Open 2016, 6, e010247. [CrossRef] [PubMed]

25. Altman, D.G.; Bland, J.M. How to Obtain the Confidence Interval from a P Value. BMJ 2011, 343, d2090. [CrossRef] [PubMed]

26. Wells, G.; Shea, B.; O'Connell, D.; Peterson, J.; Welch, V.; Losos, M.; Tugwell, P. The Newcastle-Ottawa Scale (NOS) for Assessing the Quality of Nonrandomized Studies in Meta-Analyses. Available online: http://www.ohri.ca/programs/clinical_ epidemiology / oxford.asp (accessed on 12 December 2020).

27. Huguet, A.; Hayden, J.A.; Stinson, J.; McGrath, P.J.; Chambers, C.T.; Tougas, M.E.; Wozney, L. Judging the Quality of Evidence in Reviews of Prognostic Factor Research: Adapting the GRADE Framework. Syst. Rev. 2013, 2, 1-12. [CrossRef] [PubMed]

28. Shinto, E.; Hase, K.; Hashiguchi, Y.; Sekizawa, A.; Ueno, H.; Shikina, A.; Kajiwara, Y.; Kobayashi, H.; Ishiguro, M.; Yamamoto, J. CD8+ and FOXP3+ Tumor-Infiltrating T Cells Before and After Chemoradiotherapy for Rectal Cancer. Ann. Surg. Oncol. 2014, 21, 414-421. [CrossRef] [PubMed]

29. Shinto, E.; Omata, J.; Sikina, A.; Sekizawa, A.; Kajiwara, Y.; Hayashi, K.; Hashiguchi, Y.; Hase, K.; Ueno, H. Predictive Immunohistochemical Features for Tumour Response to Chemoradiotherapy in Rectal Cancer. BJS Open 2020, 4, 301-309. [CrossRef] [PubMed]

30. Matsutani, S.; Shibutani, M.; Maeda, K.; Nagahara, H.; Fukuoka, T.; Nakao, S.; Hirakawa, K.; Ohira, M. Significance of TumorInfiltrating Lymphocytes before and after Neoadjuvant Therapy for Rectal Cancer. Cancer Sci. 2018, 109, 966-979. [CrossRef]

31. Zaghloul, H.; Abbas, A.; Abdulah, D. Tumor Microenvironment Mediators CD8+- and FOXP3+-Labeled T Lymphocytes Are Prospective Prognosticators in Curatively Treated Rectal Cancer Patients. J. Gastrointest. Cancer 2020, 52, 177-186. [CrossRef]

32. Zhang, S.; Bai, W.; Tong, X.; Bu, P.; Xu, J.; Xi, Y. Correlation between Tumor Microenvironment-associated Factors and the Efficacy and Prognosis of Neoadjuvant Therapy for Rectal Cancer. Oncol. Lett. 2019, 17, 1062-1070. [CrossRef]

33. Akiyoshi, T.; Tanaka, N.; Kiyotani, K.; Gotoh, O.; Yamamoto, N.; Oba, K.; Fukunaga, Y.; Ueno, M.; Mori, S. Immunogenomic Profiles Associated with Response to Neoadjuvant Chemoradiotherapy in Patients with Rectal Cancer. Br. J. Surg. 2019, 106, 1381-1392. [CrossRef] [PubMed]

34. Chen, T.-W.; Huang, K.C.-Y.; Chiang, S.-F.; Chen, W.T.-L.; Ke, T.-W.; Chao, K.S.C. Prognostic Relevance of Programmed Cell Death-Ligand 1 Expression and CD8+ TILs in Rectal Cancer Patients before and after Neoadjuvant Chemoradiotherapy. J. Cancer Res. Clin. Oncol. 2019, 145, 1043-1053. [CrossRef] [PubMed]

35. Malekzadeh Moghani, M.; Alahyari, S.; Moradi, A.; Nasiri, M. Pathological Predictors of Response to Neoadjuvant Treatment in Rectal Carcinoma. J. Gastrointest. Cancer 2020, 52, 690-695. [CrossRef] [PubMed]

36. Xiao, B.; Peng, J.; Zhang, R.; Xu, J.; Wang, Y.; Fang, Y.; Lin, J.; Pan, Z.; Wu, X. Density of CD8+ Lymphocytes in Biopsy Samples Combined with the Circulating Lymphocyte Ratio Predicts Pathologic Complete Response to Chemoradiotherapy for Rectal Cancer. Cancer Manag. Res. 2017, 9, 701-708. [CrossRef] [PubMed]

37. Huang, Y.; Lou, X.; Zhu, Y.; Wang, Y.; Zhang, L.; Liu, H.; Wang, C.; Zhan, H.; Cheng, Z.; Tan, W.; et al. Local Environment in Biopsy Better Predict the Pathological Response to Neoadjuvant Chemoradiotherapy in Rectal Cancer. Biosci. Rep. 2019, 39, 20190003. [CrossRef] 
38. Mirjolet, C.; Charon-Barra, C.; Ladoire, S.; Arbez-Gindre, F.; Bertaut, A.; Ghiringhelli, F.; Leroux, A.; Peiffert, D.; Borg, C.; Bosset, J.F.; et al. Tumor Lymphocyte Immune Response to Preoperative Radiotherapy in Locally Advanced Rectal Cancer: The LYMPHOREC Study. Oncoimmunology 2017, 7, e1396402. [CrossRef]

39. Huang, A.; Xiao, Y.; Peng, C.; Liu, T.; Lin, Z.; Yang, Q.; Zhang, T.; Liu, J.; Ma, H. 53BP1 Expression and Immunoscore Are Associated with the Efficacy of Neoadjuvant Chemoradiotherapy for Rectal Cancer. Strahlenther. Und Onkol. 2019, 196, 465-473. [CrossRef]

40. Rudolf, J.; Büttner-Herold, M.; Erlenbach-Wünsch, K.; Posselt, R.; Jessberger, J.; Haderlein, M.; Hecht, M.; Hartmann, A.; Fietkau, R.; Distel, L. Regulatory T Cells and Cytotoxic T Cells Close to the Epithelial-Stromal Interface Are Associated with a Favorable Prognosis. Oncoimmunology 2020, 9, 1746149. [CrossRef]

41. El Sissy, C.; Kirilovsky, A.; van den Eynde, M.; Muşină, A.-M.; Anitei, M.-G.; Romero, A.; Marliot, F.; Junca, A.; Doyen, J.; Mlecnik, B.; et al. A Diagnostic Biopsy-Adapted Immunoscore Predicts Response to Neoadjuvant Treatment and Selects Patients with Rectal Cancer Eligible for a Watch-and-Wait Strategy. Clin. Cancer Res. 2020, 26, 5198-5207. [CrossRef]

42. Apetoh, L.; Ghiringhelli, F.; Tesniere, A.; Obeid, M.; Ortiz, C.; Criollo, A.; Mignot, G.; Maiuri, M.C.; Ullrich, E.; Saulnier, P.; et al. Toll-like Receptor 4-Dependent Contribution of the Immune System to Anticancer Chemotherapy and Radiotherapy. Nat. Med. 2007, 13, 1050-1059. [CrossRef]

43. Lee, Y.; Auh, S.L.; Wang, Y.; Burnette, B.; Wang, Y.; Meng, Y.; Beckett, M.; Sharma, R.; Chin, R.; Tu, T.; et al. Therapeutic Effects of Ablative Radiation on Local Tumor Require CD8+ T Cells: Changing Strategies for Cancer Treatment. Blood 2009, 114, 589-595. [CrossRef] [PubMed]

44. Vacchelli, E.; Galluzzi, L.; Fridman, W.H.; Galon, J.; Sautès-Fridman, C.; Tartour, E.; Kroemer, G. Trial Watch. OncoImmunology 2012, 1, 179-188. [CrossRef] [PubMed]

45. Frey, B.; Rubner, Y.; Kulzer, L.; Werthmöller, N.; Weiss, E.-M.; Fietkau, R.; Gaipl, U.S. Antitumor Immune Responses Induced by Ionizing Irradiation and Further Immune Stimulation. Cancer Immunol. Immunother. 2013, 63, 29-36. [CrossRef] [PubMed]

46. Lugade, A.A.; Moran, J.P.; Gerber, S.A.; Rose, R.C.; Frelinger, J.G.; Lord, E.M. Local Radiation Therapy of B16 Melanoma Tumors Increases the Generation of Tumor Antigen-Specific Effector Cells That Traffic to the Tumor. J. Immunol. 2005, 174, 7516-7523. [CrossRef]

47. Lugade, A.A.; Sorensen, E.W.; Gerber, S.A.; Moran, J.P.; Frelinger, J.G.; Lord, E.M. Radiation-Induced IFN- $\gamma$ Production within the Tumor Microenvironment Influences Antitumor Immunity. J. Immunol. 2008, 180, 3132-3139. [CrossRef] [PubMed]

48. Topalian, S.L.; Taube, J.M.; Pardoll, D.M. Neoadjuvant Checkpoint Blockade for Cancer Immunotherapy. Science 2020, 367, eaax0182. [CrossRef]

49. Joseph, E.L.M.; Kirilovsky, A.; Lecoester, B.; El Sissy, C.; Boullerot, L.; Rangan, L.; Marguier, A.; Tochet, F.; Dosset, M.; Boustani, J.; et al. Chemoradiation Triggers Antitumor Th1 and Tissue Resident Memory-Polarized Immune Responses to Improve Immune Checkpoint Inhibitors Therapy. J. Immunother. Cancer 2021, 9, e002256. [CrossRef]

50. Andersson, P.; Ostheimer, C. Editorial: Combinatorial Approaches to Enhance Anti-Tumor Immunity: Focus on Immune Checkpoint Blockade Therapy. Front. Immunol. 2019, 10, 2083. [CrossRef]

51. Rahma, O.E.; Yothers, G.; Hong, T.S.; Russell, M.M.; You, Y.N.; Parker, W.; Jacobs, S.A.; Colangelo, L.H.; Lucas, P.C.; Gollub, M.J.; et al. Use of Total Neoadjuvant Therapy for Locally Advanced Rectal Cancer: Initial Results From the Pembrolizumab Arm of a Phase 2 Randomized Clinical Trial. JAMA Oncol. 2021, 7, 1225-1230. [CrossRef]

52. Galon, J.; Bruni, D. Tumor Immunology and Tumor Evolution: Intertwined Histories. Immunity 2020, 52, 55-81. [CrossRef]

53. Galon, J.; Bruni, D. Approaches to Treat Immune Hot, Altered and Cold Tumours with Combination Immunotherapies. Nat. Rev. Drug Discov. 2019, 18, 197-218. [CrossRef] [PubMed]

54. Bettoni, F.; Masotti, C.; Habr-Gama, A.; Correa, B.R.; Gama-Rodrigues, J.; Vianna, M.R.; Vailati, B.B.; Julião, G.P.S.; Fernandez, L.M.; Galante, P.A.; et al. Intratumoral Genetic Heterogeneity in Rectal Cancer: Are Single Biopsies Representative of the Entirety of the Tumor? Ann. Surg. 2017, 265, e4-e6. [CrossRef] [PubMed]

55. van den Eynde, M.; Mlecnik, B.; Bindea, G.; Pagè, F.; Machiels, J.-P. The Link between the Multiverse of Immune Microenvironments in Metastases and the Survival of Colorectal Cancer Patients. Cancer Cell 2018, 34, 1012-1026. [CrossRef]

56. Mlecnik, B.; Bifulco, C.; Bindea, G.; Marliot, F.; Lugli, A.; Lee, J.J.; Zlobec, I.; Rau, T.T.; Berger, M.D.; Nagtegaal, I.D.; et al. Multicenter International Society for Immunotherapy of Cancer Study of the Consensus Immunoscore for the Prediction of Survival and Response to Chemotherapy in Stage III Colon Cancer. J. Clin. Oncol. 2020, 38, 3638-3651. [CrossRef] [PubMed]

57. Pagès, F.; Mlecnik, B.; Marliot, F.; Bindea, G.; Ou, F.S.; Bifulco, C.; Lugli, A.; Zlobec, I.; Rau, T.T.; Berger, M.D.; et al. International Validation of the Consensus Immunoscore for the Classification of Colon Cancer: A Prognostic and Accuracy Study. Lancet 2018, 391, 2128-2139. [CrossRef]

58. Chetty, R.; Gill, P.; Govender, D.; Bateman, A.; Chang, H.J.; Deshpande, V.; Driman, D.; Gomez, M.; Greywoode, G.; Jaynes, E.; et al. International Study Group on Rectal Cancer Regression Grading: Interobserver Variability with Commonly Used Regression Grading Systems. Hum. Pathol. 2012, 43, 1917-1923. [CrossRef] [PubMed]

59. Westerhoff, M.; Osecky, M.; Langer, R. Varying Practices in Tumor Regression Grading of Gastrointestinal Carcinomas after Neoadjuvant Therapy: Results of an International Survey. Mod. Pathol. 2019, 33, 676-689. [CrossRef] 
60. Loughrey, M.; Quirke, P.; Shepherd, N. Dataset for Histopathological Reporting of Colorectal Cancer; Version 4; The Royal College of Pathologists: London, UK, 2018.

61. Loughrey, M.B.; Webster, F.; Arends, M.J.; Brown, I.; Burgart, L.J.; Cunningham, C.; Flejou, J.-F.; Kakar, S.; Kirsch, R.; Kojima, M.; et al. Dataset for Pathology Reporting of Colorectal Cancer. Ann. Surg. 2021; Preprint. [CrossRef] 